\title{
Differential Regulation of Immediate-Early Gene Expression in the Prefrontal Cortex of Rats with a High vs Low Behavioral Response to Methamphetamine
}

\author{
Paul D Shilling', Ronald Kuczenski', David S Segal', Thomas B Barrett ${ }^{1,2}$ and John R Kelsoe*,1,2 \\ 'Department of Psychiatry, University of California, San Diego, CA, USA; 'Department of Psychiatry, San Diego VA Healthcare System, La Jolla, \\ CA, USA
}

\begin{abstract}
Methamphetamine (METH) administration mimics many of the symptoms of mania and can produce psychosis after chronic use. Both rodents and man display interindividual variation in response to $\mathrm{METH}$. The molecular mechanisms underlying these differences might be relevant to both stimulant addiction and endogenous psychosis. We treated 50 Sprague-Dawley rats acutely with METH (4.0 mg/kg) and 10 control rats with saline, and measured their behavior for $3 \mathrm{~h}$ after drug administration. Animals were divided into high responders (HR) (top 20\%) and low responders (LR) (lowest 20\%) based on their stereotypy response. They were killed $24 \mathrm{~h}$ after injection. Total RNA was extracted from the prefrontal cortex (PFC) and the expression of approximately 30000 transcripts were analyzed using Affymetrix 2302.0 GeneChips. Real-time reverse transcription-polymerase chain reaction was used to validate the expression of a select group of genes. Forty-three genes exhibited significant differences in expression in HR vs LR $24 \mathrm{~h}$ after METH treatment including a group of immediate-early genes (IEGs) (eg, c-fos, junB, NGFI-B, serum-regulated glucocorticoid kinase). These IEG expression differences were accompanied by the significant downregulation of many of these genes compared to saline in the HR but not LR, suggesting a differential responsiveness of signal transduction pathways in these two groups of rats. In addition, the expression of other transcription factors in the PFC was significantly different in HR compared to LR. These gene expression changes may contribute to individual differences in responsiveness to stimulants and the development of mania and psychosis.

Neuropsychopharmacology (2006) 31, 2359-2367. doi:I0.1038/sj.npp. I 301 I62; published online 19 July 2006
\end{abstract}

Keywords: psychosis; mania; microarray; stereotypy; stimulant; animal model

\section{INTRODUCTION}

The mechanisms that contribute to the response to methamphetamine (METH) may be relevant to a variety of psychiatric disorders. Acute METH treatment in humans produces many symptoms of mania, including euphoria, increased energy, irritability, racing thoughts, rapid speech, hyperactivity, hypersexuality, decreased need for sleep, and psychomotor agitation (Fibiger, 1991). Acute METH treatment may, therefore, be a useful model of mania. Chronic METH use commonly results in psychotic symptoms such as paranoid delusions and hallucinations (Segal and Schuckit, 1983). For these reasons, it has been argued that acute METH may model mania and chronic METH the evolution of mania into psychotic mania. Therefore, the

\footnotetext{
*Correspondence: Dr JR Kelsoe, Department of Psychiatry, University of California, San Diego, 0603, 9500 Gilman Drive, La Jolla, CA 92093, USA, Tel: + I 858534 5927, Fax: + I 858534 5527,

E-mail: jkelsoe@ucsd.edu

Received 19 January 2006; revised 4 May 2006; accepted 9 May 2006 Online publication: 9 June 2006 at http://www.acnp.org/citations/ Npp060906060024/default.pdf
}

mechanisms involved in the response to METH may be similar to those in endogenous psychoses.

There is considerable variation among individuals in the sensitivity to stimulants such as METH (Alessi et al, 2003). Vulnerability to stimulant addiction has been shown to have heritable as well as environmental components (Saxon et al, 2005). Similarly, vulnerability to mania and psychosis has a strongly heritable basis and interindividual variation (Sklar, 2002). The molecular mechanisms underlying individual variation in response to METH may, therefore, be of relevance to both stimulant dependence and bipolar disorder and psychosis. There is presently only a limited understanding of these genetic mechanisms in man. Information from animal models regarding genes and pathways of possible relevance would be invaluable.

Similar to humans, there are also large variations in the behavioral and neurochemical responses to stimulants in rodents. For example, we have previously reported that Sprague-Dawley rats that received a moderate dose of amphetamine (AMPH) $(1.75 \mathrm{mg} / \mathrm{kg})$ could be divided into two distinct behavioral subgroups (Segal and Kuczenski, 1987). One group, low responders (LR), 
exhibited continuous locomotion usually characteristic of lower doses, whereas the other group, high responders (HR), exhibited a multiphasic pattern, which included prolonged focused stereotypy more typically associated with higher doses. These data are consistent with a subset of the animals exhibiting a greater sensitivity to the drug.

We have also observed that animals that receive high AMPH doses (greater than $2.0 \mathrm{mg} / \mathrm{kg}$, subcutaneous) exhibit variations in the onset and duration of the stereotypy and poststereotypy hyperactivity phases (Segal and Schuckit, 1983). Qualitative individual differences in the types of stereotypy are also exhibited (focused sniffing, repetitive head/limb, or oral movements). These data are consistent with differences in individual sensitivity to the stereotypyproducing effects of stimulants in rats. Large individual differences in the behavioral response to stimulants demonstrate the importance of characterizing individual response profiles in order to determine accurately the mechanisms underlying behaviors produced by METH treatment.

Measurement of gene expression in rat brain provides a powerful means to address the molecular mechanisms underlying individual responsiveness to stimulants and there is a large literature on stimulant-induced gene expression changes in rodent brain (reviewed by Harlan and Garcia, 1998; Yuferov et al, 2005). However, for the most part, these studies have not addressed individual differences. In this respect, Palmer et al (2005) recently identified gene expression differences between mice with different sensitivities to METH treatment. In addition, although many genes and pathways that are altered by stimulant treatment have been discovered, it is likely that additional important changes could have been disguised by the large individual variation in response to these drugs. The power of DNA microarrays to discover novel stimulant-regulated pathways could be augmented by applying this technology to the analysis of individual differences in gene expression in relevant brain regions.

To gain a better understanding of the mechanisms associated with the differences in the individual response to METH, and thereby, further develop a list of candidate genes and pathways of possible relevance to human stimulant dependence, mania, and psychosis, we conducted the present study. Rats were treated acutely with either METH or saline and their behavior measured. METHtreated rats were divided into $H R$ and LR based on their stereotypy response. Animals were killed $24 \mathrm{~h}$ later. This time point was selected in order to examine adaptive changes that could also be relevant to chronic treatment as the behavioral response is significantly altered after a second dose at this time point (Segal and Kuczenski, 1987). The 24-h time point also allowed us to interrogate changes that might underlie these adaptations to treatment. We measured mRNA expression in the prefrontal cortex (PFC) as evidence is consistent with this region contributing to the expression of stereotypy (Lipska et al, 1998), and changes in this brain region are also relevant to severe psychiatric disorders (Lewis et al, 2005). Affymetrix Genechips containing approximately 30000 transcripts were used to compare gene expression between HR and LR, and between these two groups and saline control.

\section{METHODS}

\section{Subjects}

Sixty male Sprague-Dawley rats $(325 \mathrm{~g})$ obtained from Simonsen laboratories (Gilroy, CA), were housed for 2 weeks before experimental manipulation in groups of two or three in wire mesh cages, with ad libitium access to food and water, in a temperature- and humidity-controlled room. The room as well as the experimental chambers were maintained on a reversed 12-h dark (0700-1900), 12-h light cycle to facilitate testing during the normal activity phase of the awake/sleeping cycle. During the dark period, all facilities were illuminated with red light to enable accurate observation of the animals. All studies adhered to animal welfare guidelines (Principles of Laboratory Animal Care, NIH Publication no. 85-23).

\section{Drugs}

D-METH hydrochloride (Sigma Chemical Co., St Louis, MO) was dissolved in saline and administered subcutaneously (s.c.) $(2 \mathrm{ml} / \mathrm{kg})$. Doses represent the free base.

\section{Behavior}

Behavior was monitored in custom-designed activity chambers. Please see Segal and Kuczenski (1997) for a detailed description of the behavioral methods and measurement of stereotypy. Raters who were unaware of the specific experimental conditions rated the videotapes on the basis of behavior ethograms and rating procedures established previously. Stereotypy and other behaviors were assessed as the percentage of the observation interval during which the animal engaged in the specific behavior and/or the number of times the behavior was displayed. The magnitude of the stereotypy response was also evaluated in terms of the onset of focused stereotypies and the duration of the focused stereotypy phase. Subjects were divided into HR (top 20\%) and LR (lowest 20\%) based on these criteria.

\section{General Procedures}

Three days before the beginning of drug administration, animals were placed in individual experimental chambers, where they remained for the duration of the experiment. To habituate animals to the procedures, they were handled and injected with saline once a day. Fifty rats were injected s.c. with $4.0 \mathrm{mg} / \mathrm{kg} \mathrm{METH}$ and an additional 10 rats were injected with saline. This experiment was designed to optimize the detection of gene expression differences between HR and LR. To decrease the variation between HR and LR, all METH-treated rats were injected before the saline-treated animals. Because this approach may have introduced the confound of detecting circadian variation in gene expression between METH-treated and saline-treated rats, we have only reported $\mathrm{METH}$-induced changes in expression for genes that exhibit significant differences between HR and LR. Behavior was measured for $3 \mathrm{~h}$ after injection as described previously. Twenty-four hours after injection, all rats were killed, brains removed, and the PFC was dissected and quick frozen in liquid nitrogen and stored at $-80^{\circ} \mathrm{C}$. 


\section{GeneChip experiments}

Total RNA was isolated from tissue using RNeasy columns (Qiagen, Valencia, CA). RNA quality was checked by Agilent Lab-on-a-chip and spectrophotometry (260/280). Total RNA $(5 \mu \mathrm{g})$ was used to prepare cDNA. cDNA synthesis, cRNA amplification, hybridization to Affymetrix 2302.0 Genechips, and subsequent washes and scanning were performed according to the Affymetrix standard protocols (http://www.affymetrix.com/support/technical/manuals.affx). The cRNA from individual animals was hybridized to each GeneChip.

\section{DNA Microarray Data Analysis}

Raw images were analyzed and features extracted using GCOS 1.1 (Affymetrix, Foster City, CA). The resulting CEL files containing probe level information were then normalized and converted to gene intensity values by the Robust Multi-Array Average algorithm (Irizarry et al, 2003). Statistical analyses were performed on these normalized values for the saline, $H R$, and LR groups by ANOVA followed by Student-Neuman-Keuls (SNK) post hoc tests (Genespring, Agilent Technologies, Palo Alto, CA). Significant gene expression differences between $\mathrm{HR}$ and LR detected by ANOVA $(p<0.01)$ and significantly different by SNK post hoc analysis $(p<0.05)$ are described in this paper. We chose an ANOVA nominal $p<0.01$ as an arbitrary cutoff to detect the most robust gene expression differences. No genes survived statistical analyses that corrected for multiple comparisons of the 30000 transcripts. Genes exhibiting HR/LR or LR/HR ratios $<1.1$ were excluded. GeneSpring refers to these ratios as fold change. Expression differences were based on fold change calculated as the average expression over all samples in condition 1 divided by the average expression over all samples in condition 2. Both HR/LR and LR/HR ratios were used to determine the exclusion criterion as neither group served as a baseline comparator. Hierarchical Cluster Analysis (GeneSpring) was performed on those genes that exhibited significant expression differences between $H R$ and LR to identify similar expression patterns. MetaCore GeneGo (St Joseph, MI) was used to identify pathways and networks. Database for Annotation, Visualization, and Integrated Discovery (DAVID) (National Institute of Allergy and Infectious Diseases) and NETAFFX (Affymetrix) were used to annotate genes.

\section{Taqman Reverse Transcription-Polymerase Chain Reaction (RT-PCR)}

First-strand cDNA was synthesized using SuperScript III (Invitrogen, Carlsbad, CA). For each sample, $1 \mu \mathrm{g}$ of total cellular RNA was reverse transcribed in a $20 \mu \mathrm{l}$ reaction with $50 \mathrm{ng}$ of random hexamer primers and RNaseOUT according to the manufacturer's protocol. cDNAs were then diluted 1:7 with $\mathrm{H}_{2} \mathrm{O}$ and $7 \mu \mathrm{l}$ were assayed for level of RNA expression by real-time PCR using Applied Biosystems' TaqMan Gene Expression Assays performed on an Applied Biosystems 7300 in a $20 \mu$ reaction with Universal PCR Master Mix (without AmpErase ${ }^{\circledR}$ UNG) according to the manufacturer's protocol. Genes and assay ID numbers were as follows: for serum-regulated glucocorticoid kinase (Sgk), Rn00570285; for activity-regulated cytoskeletal protein (Arc), Rn00571208_g1; for beta-2-microglobulin (B2M), Rn00560865_m1; for ribosomal protein S6 (RPS6), Rn00820815_g1; for c-fos a custom Assay-by-Design was used. Each assay was performed in triplicate, $C_{\mathrm{t}}$ values were converted to a linear scale, and the mean for each triplicate was determined. Two 'unregulated' genes, RPS6 and B2M, were selected for normalization based on their having minimal variance across all animals as detected by the Affymetrix chip. The RT-PCR values for these two controls were equally weighted and combined by adjusting the mean linear value of all samples for each control gene to 1.0 and then averaging the two values for each cDNA sample. The result for each experimental triplicate was then normalized to this corresponding weighted control.

\section{RESULTS}

\section{Behavior}

The temporal pattern of locomotor activation induced by $4 \mathrm{mg} / \mathrm{kg}$ of METH administration was consistent with our previous data (Segal and Kuczenski, 1997). Rats exhibited a triphasic response consisting of early and late periods of primarily locomotion and an intermediate phase of continuous focused oral stereotypy (Figure 1). Individual behavioral profiles were divided into $\mathrm{HR}$ and LR based on their stereotypy response to METH. HR exhibited a stereotypy response to METH in the upper $20 \%$ of the rats screened and LR in the bottom $20 \%$. Compared to the LR, the HR displayed a decreased latency to intense focused oral stereotypy (30 vs $40 \mathrm{~min}$ postinjection) and a longer duration of focused oral stereotypy $(140$ vs $80 \mathrm{~min}$ postinjection). The stereotypy data approximated a normal distribution. The results in each group were qualitatively similar in that (1) the multiphasic shape of the locomotor response was comparable and, (2) all animals exhibited predominantly oral behaviors and repetitive head and limb movements.

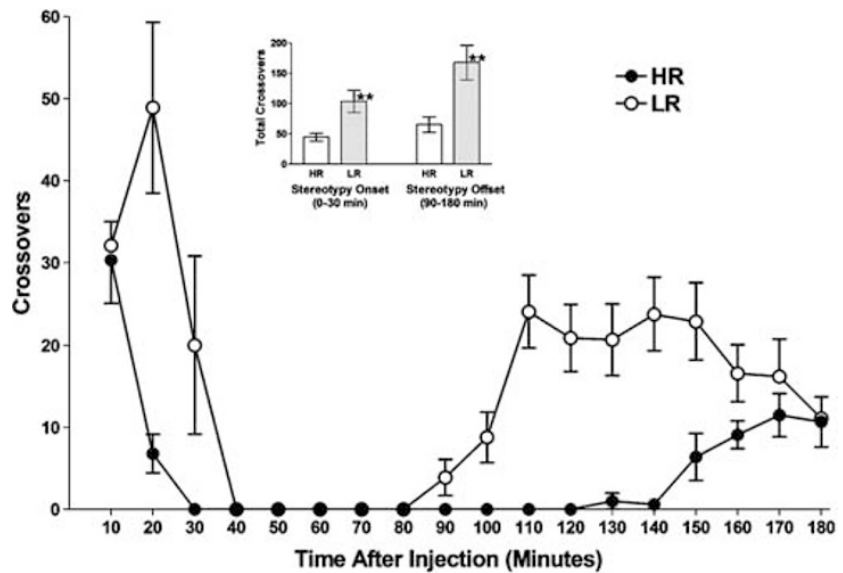

Figure I (Main) The temporal pattern of locomotor activation induced by $4 \mathrm{mg} / \mathrm{kg}$ of METH administration. Individual behavioral profiles were divided into $H R$ and $L R$ based on their stereotypy response to METH treatment. (Inset) Total crossovers exhibited during stereotypy onset and offset, $H R$ vs LR. *** Significantly different from $H R, p<0.01$. 
In addition, consistent with their higher levels of stereotypy, the HR exhibited significantly decreased crossovers compared to LR for the first $30 \mathrm{~min}(t(18)=3.3$, $p<0.01$ ), corresponding to the more rapid onset of focused stereotypies and $90-180 \mathrm{~min}(t(18)=3.0, p<0.01)$ after METH administration, corresponding to a longer duration of stereotyped behaviors (Figure 1, inset).

\section{Gene Expression Analyses}

We performed several methods of quality control to confirm that all RNA probes and DNA chips were of the highest quality. In this regard, the $3^{\prime}: 5^{\prime}$ ratio expression signal for $3^{\prime}$ probes compared to $5^{\prime}$ probes for the housekeeping gene glyceraldehyde-3-phosphate dehydrogenase (GAPDH) was $<1.21$ and $>0.91$ for all samples, indicating a high level of transcription across the entire transcript. In addition, for all 30 DNA chips background values were $<105$, which is considered an acceptable background hybridization (Affymetrix).

\section{Differential Gene Expression-HR vs LR}

We detected 43 genes that exhibited significant differences in expression in HR vs LR $24 \mathrm{~h}$ after METH treatment (ANOVA $p<0.01$ and significant by SNK post hoc test), with a minimum $\mathrm{HR} / \mathrm{LR}$ or $\mathrm{LR} / \mathrm{HR}$ ratio $\geqslant 1.1$. Hierarchical Cluster Analysis of these 43 genes is displayed in Figure 2. Genes that exhibited more highly correlated levels of

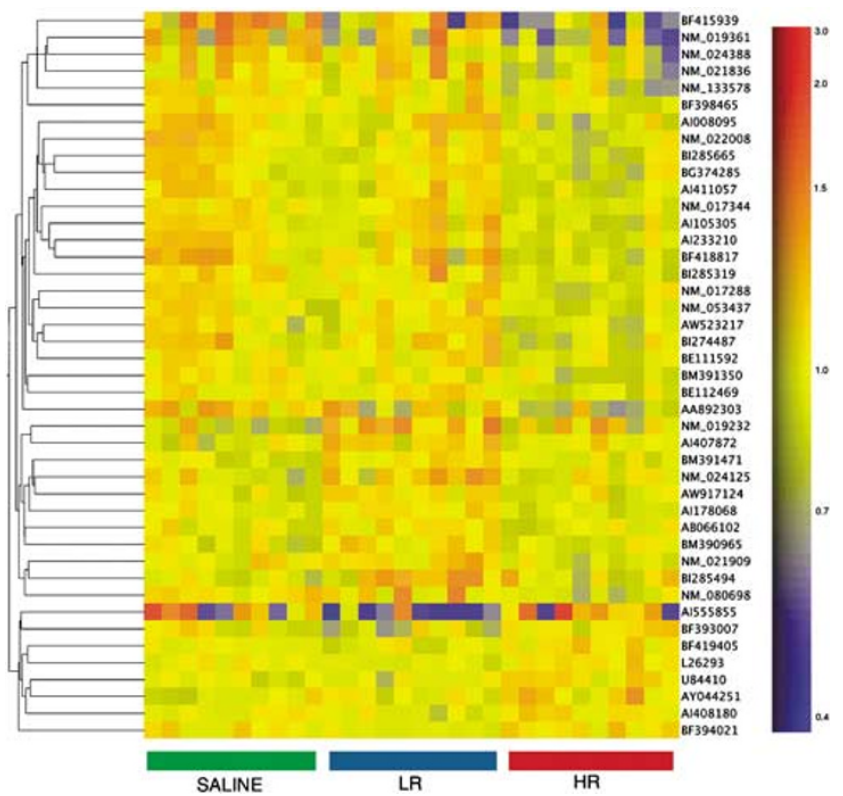

Figure 2 Hierarchical Cluster Analysis. Hierarchical clustering of genes exhibit significant expression differences between HR and LR in the PFC. Samples with similar expression patterns are grouped together in a dendrogram. All normalized expression values were renormalized to a median value of 1 . The first 10 columns represent the 10 saline-treated animals. The second 10 columns represent the LR, and the last 10 columns represent the HR. The genes are represented in each row. Red indicates higher than the median and blue less than the median gene expression values (see key). gene expression are clustered together, suggesting coregulation. Several patterns of gene expression can be delineated from this cluster analysis. However, the most robust pattern is of genes exhibiting significantly decreased expression in HR relative to LR. Genes that exhibited significant differences in expression between LR and HR are displayed in Table 1 and grouped by functional category. Categories are represented that included a minimum of two genes. The functional group that displayed the greatest changes in response to METH was immediate-early genes (IEGs). Other functional categories displaying the greatest number of changed genes included: other transcription factors, signal transduction, ion transport, and cytokine regulation. These expression differences suggest substantial adaptive changes in a variety of systems relevant to neural function.

These METH-induced changes in IEG expression including c-fos, NGFI-B, Arc, junB, MAP kinase phosphatase (Cpg21), and Sgk are displayed in Table 1 and Figure 3a. The most striking aspect of these gene changes is that for most of these genes the level of expression of the HR group is lower than that of the LR group. This is true whether the gene is upregulated in relation to saline or downregulated.

As shown in Table 1 and Figure $3 \mathrm{~b}$, transcription factors other than IEGs also displayed some of the most significant differential changes observed. In contrast to the IEGs, most of these transcription factors were upregulated in the LR but not in the HR. These factors included CAAT enhancer binding protein $\beta$, activating transcription factor 5 , zincfinger protein 189, and transcription factor IIH. Consistent with the IEGs, gene expression for most of these genes was lower in HR compared to LR.

A number of genes of relevance to signal transduction exhibited significant expression differences between HR and LR, including adenylate cyclase 5 , glycogen synthase kinase 3 alpha (Gsk-3a), diacylglycerol $O$-acyltransferase, and caspase-3. These gene expression differences are exhibited in Table 1 . Of note, for the vast majority $(81 \%)$ of the genes differentially expressed in HR $v s$ LR, the level of expression was lower in HR compared to LR.

\section{Gene Expression Differences Validated by Taqman RT-PCR}

Sgk, c-fos, and Arc were selected from the METH-induced gene expression changes for validation with Taqman RTPCR. The significant gene expression differences between SAL and LR for Sgk, and saline and HR for c-fos and Arc detected on the GeneChips were verified for all these genes $(p<0.05)$ (Figure 4). In addition, comparison of the microarray and RT-PCR expression values for each of these genes revealed a significant Pearson's correlation for each of these genes (Sgk: $r=0.71 ; c-f o s: r=0.80 ;$ Arc: $r=0.81$; $p<0.001)$. However, the significant differences detected by the DNA chips between LR and HR for Sgk, c-fos, and Arc were all detected as nonsignificant trends consistent with the direction of change by RT-PCR. These data suggest that some of these gene expression changes were too small to be reliably detected as significant differences by real-time RTPCR and are most likely owing to the greater interindividual variation in the METH-treated animals for the RT-PCR data compared to the DNA GeneChip data. 
Table I HR vs LR: Differentially Expressed Genes Represented by Functional Categories

Table I HR ws LR: Differentially Expressed Genes Represented by Functional Categories

\begin{tabular}{|c|c|c|c|c|c|}
\hline \multirow[b]{2}{*}{ Accession number } & \multirow[b]{2}{*}{ Genes } & \multirow[b]{2}{*}{ ANOVA $p$-value } & \multicolumn{3}{|c|}{ Fold change } \\
\hline & & & HR/LR & HR/SAL & LR/SAL \\
\hline & Immediate early genes & & & & \\
\hline BF4I5939 & c-fos & 0.003 & 0.7 & $0.54 *$ & 0.79 \\
\hline NM_01936 | & activity regulated cytoskeletal-associated protein & 0.007 & 0.76 & $0.65 *$ & 0.86 \\
\hline NM_021836 & JunB oncogene & 0.003 & 0.78 & $0.82 *$ & 1.06 \\
\hline NM_024388 & NGFI-B (Apoptosis) & 0.0099 & 0.85 & $0.78^{*}$ & 0.92 \\
\hline NM_I 33578 & MAP kinase phosphatase & 0.008 & 0.88 & $0.85 *$ & 0.9 \\
\hline \multirow[t]{2}{*}{ NM_019232 } & Serum-regulated glucocorticoid kinase (Apoptosis) & 0.002 & 0.84 & 1.17 & $1.39 *$ \\
\hline & Additional transcription factors & & & & \\
\hline BM39|47| & activating transcription factor 5 & 0.001 & 0.91 & 1.03 & $1.13 *$ \\
\hline NM_024I25 & CCAAT/enhancer binding protein, beta (Apoptosis) & 0.005 & 0.83 & 1.02 & $1.23 *$ \\
\hline Al407872 & zinc finger protein 189 & 0.005 & 0.9 & 1.05 & $1.17 *$ \\
\hline \multirow[t]{2}{*}{ AW917124 } & $62 \mathrm{kDa}$ subunit of transcription factor $\| \mathrm{H}$ & 0.009 & 0.9 & 0.99 & $1.10 *$ \\
\hline & Signal transduction & & & & \\
\hline Al008095 & adenylate cyclase 5 & 0.009 & 0.85 & $0.83 *$ & 0.98 \\
\hline NM_0I7344 & glycogen synthase kinase 3 alpha & 0.001 & 0.89 & $0.90 *$ & 1.0 \\
\hline U84410 & caspase 3 (Apoptosis) & 0.004 & 1.11 & $1.11 *$ & 1.0 \\
\hline \multirow[t]{2}{*}{ NM_053437 } & diacylglycerol O-acyltransferase I & 0.007 & 0.88 & $0.89 *$ & 1.02 \\
\hline & Ion transport & & & & \\
\hline NM_021909 & FXYD domain-containing ion transport regulator 5 & 0.001 & 0.88 & $0.92 *$ & 1.08 \\
\hline NM_022008 & FXYD domain-containing ion transport regulator 7 & 0.005 & 0.91 & $0.85 *$ & 0.93 \\
\hline \multirow[t]{2}{*}{ NM_017288 } & sodium channel, voltage-gated, type I, beta & 0.003 & 0.89 & $0.89 *$ & 1.0 \\
\hline & Cytokine regulation & & & & \\
\hline B1285494 & interferon induced transmembrane protein 3-like & 0.007 & 0.85 & $1.17 *$ & 1.03 \\
\hline \multirow[t]{2}{*}{ AY04425I } & interleukin 13 receptor, alpha I & 0.008 & 1.13 & 1.07 & $1.27 *$ \\
\hline & Other & & & & \\
\hline AA892303 & dynein axonemal, light chain 4 & 0.005 & 0.82 & $0.74 *$ & 0.91 \\
\hline All 05305 & WW domain binding protein 2 & 0.008 & 0.86 & $0.87 *$ & 1.02 \\
\hline Al233210 & lymphocyte antigen 6 complex & 0.007 & 0.9 & $0.85 *$ & 0.95 \\
\hline All 78068 & required for cell differentiation I & 0.0003 & 0.9 & 1.04 & $1.13^{*}$ \\
\hline AB066102 & thyroid autoantigen & 0.001 & 0.9 & 0.97 & $1.07 *$ \\
\hline L26293 & FSH-regulated protein mRNA & 0.0003 & 1.1 & $1.05 *$ & $0.96 *$ \\
\hline BM39/350 & collagen alpha $2(X \mathrm{I})$ chain precursor & 0.001 & 0.91 & $0.87 *$ & 0.96 \\
\hline BF393007 & T-cell receptor alpha/delta 4 & 0.006 & 1.19 & $0.89 *$ & 1.06 \\
\hline Al41 1057 & coactosin-like | & 0.008 & 0.88 & $0.86 *$ & 0.97 \\
\hline NM_080698 & fibromodulin & 0.0096 & 0.81 & $0.86 *$ & 1.07 \\
\hline Al555855 & EST homology to human nuclear factor $1 / X$ & 0.007 & 1.91 & 1.03 & $0.52 *$ \\
\hline Al408|80 & EST homology to TAF6-like RNA polymerase ॥ & 0.002 & 1.1 & $1.05 *$ & 0.96 \\
\hline B1285319 & EST homology to mouse cecum & 0.006 & 0.85 & $0.88^{*}$ & 1.04 \\
\hline B1285665 & EST homology to mouse tweety homolog 2 & 0.005 & 0.91 & $0.87 *$ & 0.96 \\
\hline AW523217 & EST homology to human suppressor of cytokine 4 & 0.005 & 0.88 & $0.86 *$ & 0.98 \\
\hline B1274487 & EST homology to tissue inhibitor of metalloproteinase 2 & 0.0099 & 0.85 & $0.86 *$ & 1.01 \\
\hline BF4 18817 & EST & 0.002 & 0.80 & $0.76 *$ & 0.98 \\
\hline BM390965 & EST & 0.006 & 0.9 & 1.01 & $1.12 *$ \\
\hline BEIII592 & EST & 0.008 & 0.9 & $0.89 *$ & 0.99 \\
\hline BF39402I & EST & 0.007 & 1.1 & 1.04 & $0.94 *$ \\
\hline BEI I 2469 & EST & 0.005 & 0.91 & $\left.0.9\right|^{*}$ & 1.01 \\
\hline BF398465 & EST & 0.009 & 0.85 & $0.89 *$ & 0.99 \\
\hline BF4I9405 & EST & 0.005 & 1.11 & $1.07 *$ & 0.97 \\
\hline
\end{tabular}

\footnotetext{
*Significantly different from saline (SAL) $(p<0.05)$.
} 

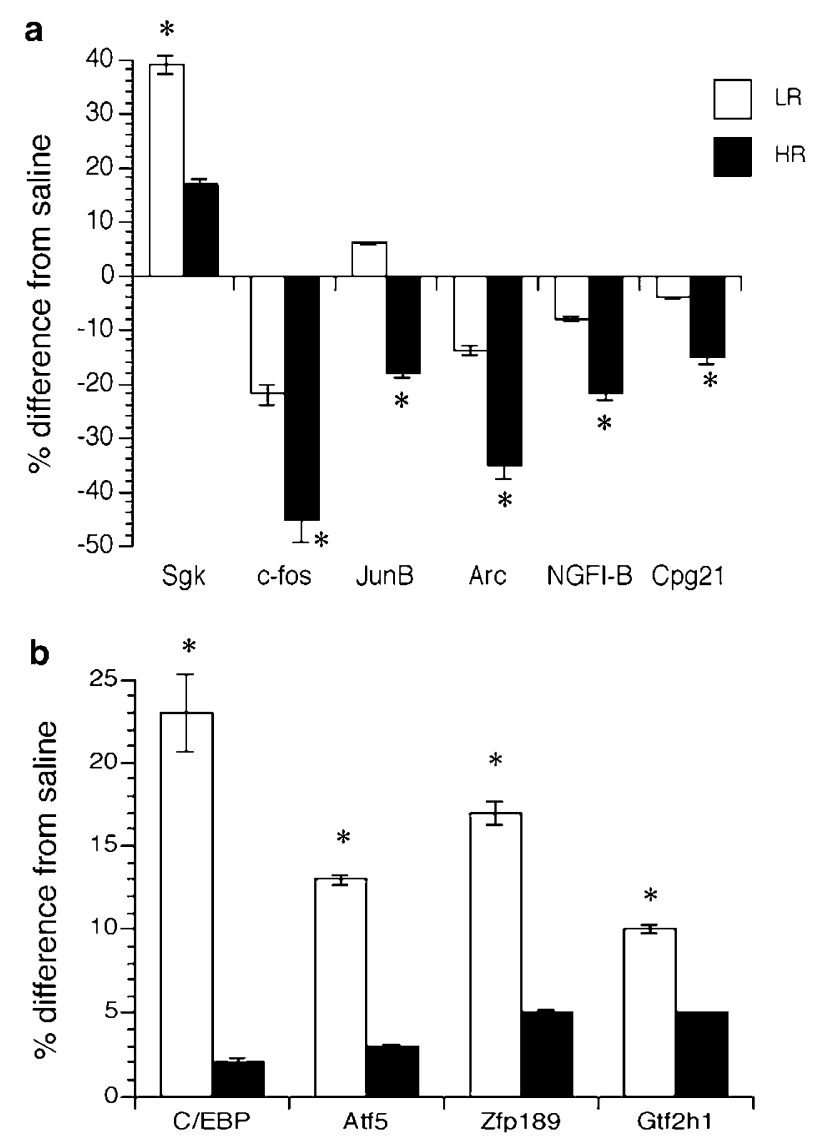

Figure 3 (a) Effects of METH in HR vs LR on the expression of the IEGs: serum-regulated glucorticoid kinase (Sgk), c-fos, junB, activity regulated cytoskeletal protein (Arc), NGFI-B and MAP kinase phosphatase (Cpg2I), $24 \mathrm{~h}$ after injection. (b) The effects of METH in HR vs LR on additional transcriptional regulators: CAAT enhancer-binding protein $\beta$ (C/EBP), activating transcription factor 5 (Atf5), zinc-finger protein (Zfp) 189, and transcription $\mathrm{IH}(\mathrm{Gtf2fI}) 24 \mathrm{~h}$ after injection. Expression differences between $H R$ and $L R$ are significant $(p<0.05)$ for all genes represented. *Significantly different from saline, $p<0.05$. Values represent the mean percentage difference from saline control \pm SEM.

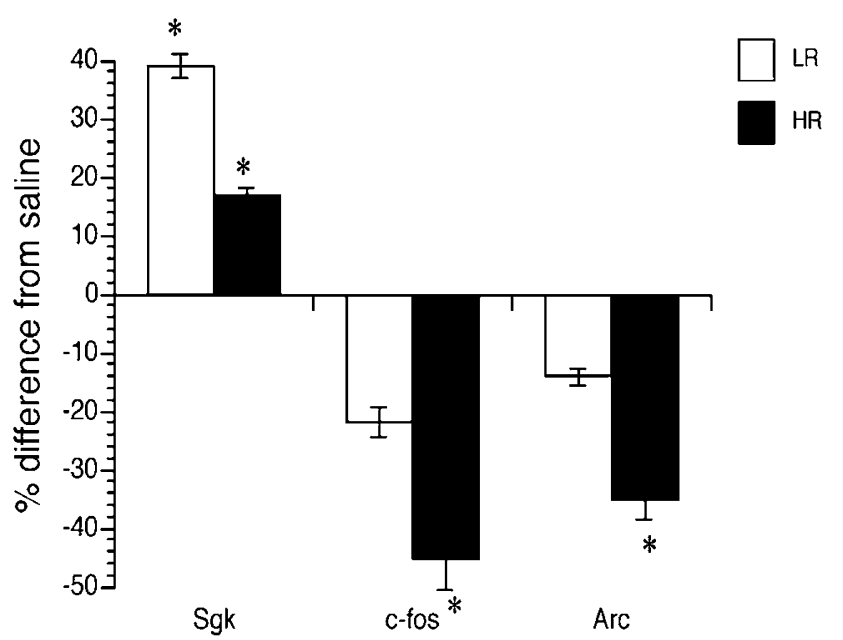

Figure 4 Real-time RT-PCR validation. Real-time RT-PCR for Sgk, c-fos, and Arc in $H R$ and $L R$. *Significantly different from saline $(p<0.05)$. Expression values were normalized to $\beta$-2-microglobulin and ribosomal protein $\mathrm{S6}$. Values represent the mean percentage difference from saline control \pm SEM.

\section{DISCUSSION}

To identify gene expression and pathways that respond differently to METH treatment in HR compared to LR, we measured the expression of approximately 30000 transcripts in the PFC $24 \mathrm{~h}$ after METH treatment. These data revealed many significant differences in gene expression between HR and LR. For example, we detected significant differences in IEG expression in HR $v s$ LR that were reflected in a significant downregulation in the HR but not in the LR compared to saline $24 \mathrm{~h}$ after METH treatment. IEGs such as c-fos are typically upregulated $1-2 \mathrm{~h}$ after stimulant treatment in several brain regions (Graybiel et al, 1990; Shilling et al, 2000; Cadet et al, 2001), a response indicative of general cell activation. Of the IEGs that have been studied over a prolonged time course including c-fos, several exhibit a significant downregulation $12-48 \mathrm{~h}$ after stimulant treatment (Ennulat et al, 1994; Persico et al, 1995), consistent with our results.

HR but not LR exhibited a significant downregulation of these genes, raising the possibility that in previous studies the enduring downregulation detected in stimulant-treated animals actually reflects individual differences in METH response that are primarily owing to IEG expression changes in HR. Many of these downregulated IEGs, such as c-fos, NGFI-B, etc, are transcription factors, which are regulated by upstream signal transduction processes. The differences in the regulation of these genes in HR and LR would suggest differences between these two groups in one or more of the upstream regulatory processes that contribute to the expression of these transcription factors. For example, activation of D1 receptors is necessary for AMPH-induced upregulation of c-fos (Konradi et al, 1994; Moratalla et al, 1996). D1 but not D2 antagonists block METH-induced upregulation of IEGs in the cortex (Yamagata et al, 2000; Glickstein and Schmauss, 2004). In addition to D1 activation, G-protein activation of cAMP and resulting CREB phosphorylation by protein kinase $A$ are necessary for AMPH regulation of $c$-fos transcription (Konradi et al, 1994). Adenylate cyclase 5 is also differentially regulated in HR $v s$ LR plays a major role in integrating D1 and D2 receptor-mediated activation of this pathway (Lee et al, 2002). This pathway is displayed in Figure 5. Furthermore, activation of D1 receptors also plays a major role in the expression of stereotypy (Chartoff et al, 2001).

Other nontranscription factor IEGs were also differentially regulated in the HR and LR, including Arc, Sgk, and Cpg21. METH-induced alterations in the expression of these genes is also consistent with previous reports (Takaki et al, 2001; Gonzalez-Nicolini and McGinty, 2002). These genes also have been reported to be upregulated shortly after stimulant administration. To the best of our knowledge, this is the first report of a lasting upregulation of Sgk and a lasting downregulation of Arc and Cpg21 after acute stimulant treatment.

The function of a lasting difference in IEG expression between HR and LR reflected in the downregulation of IEGs specifically in the HR but not LR has several possible interpretations. It has been previously suggested that these enduring changes in IEG expression could contribute to the mediation of long-term effects of 
stimulants (Ennulat et al, 1994). This possibility would suggest that METH treatment in $\mathrm{HR}$ and LR produces different long-term effects. It is possible that this downregulation represents compensatory adaptations to the initially large increases in the expression of these genes that have been consistently observed shortly after stimulant treatment. Therefore, $24 \mathrm{~h}$ after METH treatment gene expression alterations could be owing to the direct effects of the drug or adaptive changes. Although it would be difficult to differentiate these two possibilities, gene expression differences between HR and LR that are owing to either drug effects or adaptive changes would both represent individual differences in response to METH.

It is also possible that $24 \mathrm{~h}$ after METH treatment changes in the expression of IEGs (as well as other genes) are owing to the activity differences (locomotion or stereotypy) between the HR and LR. In fact, locomotor activity transiently increases the expression of several genes in the rat motor cortex and hippocampus (eg, c-fos) (Eilam et al, 1999) and could also induce gene expression in the PFC. However, it would not be possible to differentiate unequivocally the effects of METH and activity differences on gene expression under our experimental conditions.

Evidence suggests that IEGs could also play a neuroprotective role against the neurotoxic effects of METH. For example, mice lacking c-fos exhibit greater $\mathrm{METH}$-induced neurotoxicity (Betts et al, 2002). Consistent with a neuroprotective effect, several apoptosis-related genes (see Table 1) were also differentially regulated in the HR $v s$ LR. It seems plausible that as IEG expression is usually rapidly induced after various stressors and drug treatments, these genes could provide a protective response to these adversive stimuli. Consistent with this possibility, animals that are more sensitive to a drug, such as the HRs, would exhibit a greater IEG response such as the significant downregulation of many IEGs in HR but not LR. In this respect, it would be important to determine if HR also exhibit a greater increase compared to LR in IEG expression shortly after METH treatment.

Many additional transcriptional regulators also exhibited significant HR vs LR expression differences. For example, CAAT enhancer-binding protein $\beta$, which was reported previously to be upregulated by stimulants (Cadet et al, 2001; Sokolov et al, 2003), was upregulated in the LR but not HR. In addition, the differential regulation of activating transcription factor 5, a protein that alters transcription in response to changing cellular cAMP levels is consistent with different adaptations in cAMP signal transduction pathways, as are differences in the expression of adenylate cyclase 5 . These differences in the regulation of transcription factor expression also suggest that HR and LR exhibit different long-term adaptations to METH treatment. Differential changes in other functional processes represented by the differentially expressed genes such as signal transduction, ion transport, and cytokine regulation represent additional potential neural adaptations to the differences in response to METH in LR and HR.

Lithium has been reported to negatively regulate Gsk-3a (Beaulieu et al, 2004) and therefore expression changes in this pathway could be relevant to mania. In this regard, Gsk-3a was differentially expressed in HR vs LR and has previously been reported to be downregulated after stimulant administration (Yuferov et al, 2005). The downregulation of Gsk-3a in HR but not LR (Figure 5) could represent a compensatory adaptation to differences in these pathways in these two groups of rats. This protein contributes to the downstream regulation of the behavioral effects of dopamine (DA) mediated by the D2 receptor. It is thought that the synergistic effects of D2 agonists on D1mediated behaviors could occur through this signal transduction pathway that is modulated by Gsk-3 (alpha/ beta) (Beaulieu et al, 2004). Therefore, the differential expression of this gene in HR vs LR in response to METH administration could represent adaptations to differences in upstream signal transduction mechanisms between these two groups.

The relatively subtle fold changes detected in our study are consistent with previous studies using individual animals to investigate the effects of METH in the brain. In this regard, Bowyer et al (2004) found that METH treatment produced greater than three-fold changes in expression shortly after treatment. However, changes $24 \mathrm{~h}$ and longer after METH are consistent with less than two-fold changes (Bowyer et al, 2004; Ogden et al, 2004).

Of note, most of the genes (81\%) that exhibited differential expression between $\mathrm{HR}$ and $\mathrm{LR}$ tended to exhibit lower expression levels in the HR compared to LR. For downregulated genes, this expression pattern most likely reflects more enduring expression changes in HR vs LR, which could be owing to a differing time course of expression. For genes upregulated in LR but not in HR, these expression differences could also reflect a different time course of expression for these two groups of rats.

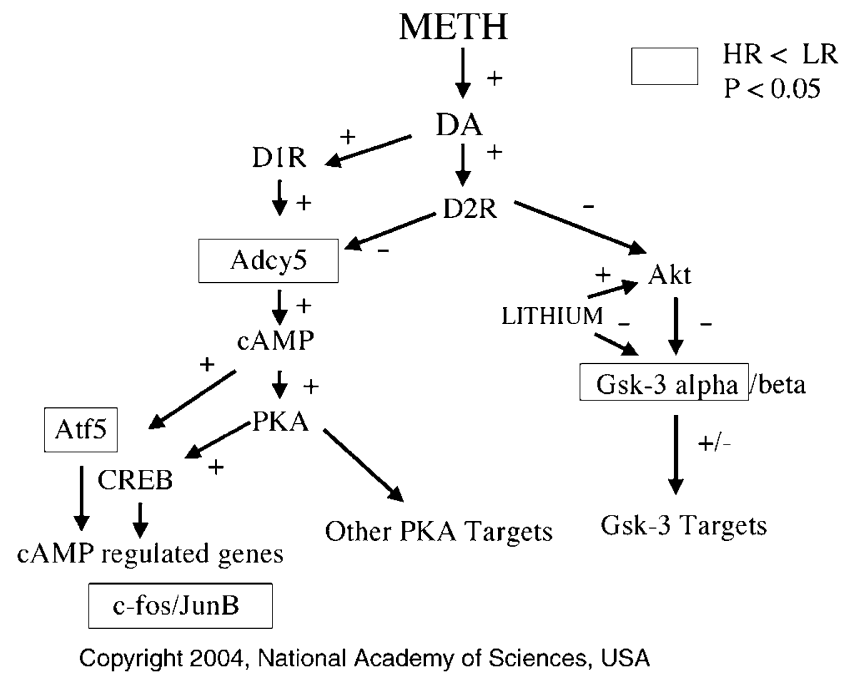

Figure 5 Working model of the differential effects of METH in HR and LR on dopaminergic signaling pathways. Dopaminergic activation of DI receptor positively regulates the CAMP pathway resulting in differential effects on downstream gene expression (eg, Adcy5, c-fos, and junB) in HR vs LR. D2 receptor activation inhibits Akt enhancing the action of Gsk-3 (alpha/beta) on its downstream targets and Gsk-3a is downregulated in the HR but not the LR. These gene expression changes suggest differential responsiveness of these signal transduction pathways and differential longterm adaptations in HR vs LR (adapted from Beaulieu et al, 2004). Abbreviations: DIR, dopamine (DA) DI receptor; D2R, dopamine (DA) D2 receptor; Adcy5, adenylate cyclase 5; Atf5, activating transcription factor 5; PKA, protein kinase A; CREB, CAMP response element-binding protein; and Gsk, glycogen synthase kinase. 
For example, at $24 \mathrm{~h}$ after METH treatment, a gene still upregulated in the LR could have been upregulated at a previous time point in the HR but at $24 \mathrm{~h}$ after treatment expression has decreased to baseline consistent with a different time course of expression. In this regard, one of the limitations of our study is that we only used one time point. Therefore, to determine if these possibilities are correct, time course studies will need to be performed.

There are a limited number of reports addressing neurochemical correlates of the individual differences in the behavioral response to stimulants. For example, Palmer et al (2005) recently reported that a number of genes were differentially expressed in the nucleus accumbens of mice bred for high and low behavioral responses to METH. None of these genes were detected as differentially regulated in HR vs LR, in our study. This is not surprising as our approach identified differences in gene expression of relevance to the variation in METH responsiveness in the PFC of rats, whereas the Palmer et al (2005) study identified gene expression differences between drug-naïve HR and LR in the nucleus accumbens of mice.

In addition, of relevance to individual differences, Segal and Kuczenski (1987) found that after a moderate dose of AMPH used to behaviorally differentiate HR from LR, HR exhibited significantly higher levels of homovanillic acid (30 min after treatment) in the frontal cortex than LR, suggesting a differential DA response in this brain region. In addition, Hooks et al (1992) found that in response to cocaine HR animals exhibited significantly higher DA levels in the nucleus accumbens compared to LR. These differences in the homovanillic acid and DA levels are consistent with D1/D2-mediated signal transduction differences in gene expression between HR and LR.

It is of great interest that several genes known to be regulated by glucocorticoids were among the genes differentially regulated in $\mathrm{HR} v s \mathrm{LR}$, for example, Sgk, c-fos, junB, NGFI-B, and Cpg21 (Umemoto et al, 1997; $\mathrm{Wu}$ et al, 2004a). All of these genes also have previously been reported to be regulated by stimulant administration. In addition, Gsk-3 signaling pathways have also been reported to be regulated by glucocorticoids (Wu et al, 2004b). As higher levels of plasma glucocorticoids are associated with increased levels of stimulant-induced stereotypy (Swerdlow et al, 1993) and glucocorticoids regulate the expression of genes induced by $\mathrm{METH}$ treatment, it seems possible that differences in responsiveness of the hypothalamic-pituitary-adrenal (HPA) axis to METH treatment could underlie some of the behavioral differences exhibited by LR vs HR.

We previously had performed a microarray study on the caudate of these animals and found a small number of significant differences between $\mathrm{HR}$ and LR that were characterized primarily by expressed sequence tags (ESTs) (data not shown). Because of the high percentage of ESTs and the lack of multiple genes represented in specific functional categories, these data were difficult to interpret. It might be expected that as the PFC is not as intimately associated with stereotypical behavior as the caudate that METH-induced gene expression, changes in this brain region may not be as relevant to these behaviors. However, as previously described, the PFC modulates stereotypy and the more robust METH-induced differences in HR vs LR gene expression in the PFC suggests a role for this brain region in individual differences in responsiveness to this stimulant.

Finally, stimulant-induced behavioral sensitization, the augmented behavioral response to repeated stimulant treatment, is seen as soon as $24 \mathrm{~h}$ after an acute METH injection. Therefore, expression differences we detected $24 \mathrm{~h}$ after METH treatment between HR and LR could also be relevant to differences in behavioral sensitization in these two groups of rats. For example, HR were reported to exhibit a different behavioral response compared to LR to repeated AMPH injection (Segal and Kuczenski, 1987), which could be owing to the different adaptations in signal transduction pathways we detected $24 \mathrm{~h}$ after METH treatment in these two groups of rats. Although it is not totally clear whether the gene expression changes we detected reflect drug effects or adaptive changes, any change detected $24 \mathrm{~h}$ after METH treatment could have relevance to sensitization.

In summary, we detected many differences in gene expression in HR compared to LR after METH treatment that could be relevant to the differences in behavioral responsiveness between these two groups. In this regard, it is likely that differentially expressed genes in $\mathrm{HR} v s \mathrm{LR}$ represent different long-term adaptations in signal transduction pathways $24 \mathrm{~h}$ after stimulant administration that could contribute not just to interindividual differences in initial responsiveness but also to the enduring effects of behavioral sensitization. Interestingly, many of the differentially expressed genes are also regulated by glucocorticoids, suggesting a role for the HPA axis in modulating some of these differences in responsiveness. Because of the many similarities in the behavioral responses in rats produced by METH treatment and mania in humans, it is possible that the gene expression differences reported here could identify genes and pathways that mediate the individual variation in susceptibility to mania, psychosis, and stimulant dependence.

\section{ACKNOWLEDGEMENTS}

This work was supported in part by grants to JRK from the NIH (MH47612, MH59567, MH68503, DA13769) and the Department of Veterans Affairs, to PDS from the MIRECC, and to RK and DSS from the NIDA DSA-01568 and DA02854, and to TBB from the NIMH MH067959 and MH71911. JRK and TBB are founders and hold equity in Psynomics, Inc. We thank Skip McCunney for his excellent technical assistance.

\section{REFERENCES}

Alessi SM, Greenwald M, Johanson CE (2003). The prediction of individual differences in response to D-amphetamine in healthy adults. Behav Pharmacol 14: 19-32.

Beaulieu JM, Sotnikova TD, Yao WD, Kockeritz L, Woodgett JR, Gainetdinov RR et al (2004). Lithium antagonizes dopaminedependent behaviors mediated by an AKT/glycogen synthase kinase 3 signaling cascade. Proc Natl Acad Sci USA 101: 5099-5104.

Betts ES, Krasnova IN, McCoy MT, Ladenheim B, Cadet JL (2002). Analysis of methamphetamine-induced changes in the 
expression of integrin family members in the cortex of wild-type and c-fos knockout mice. Neurotox Res 4: 617-623.

Bowyer JF, Harris AJ, Delongchamp RR, Jakab RL, Miller DB, Little AR et al (2004). Selective changes in gene expression in cortical regions sensitive to amphetamine during the neurodegenerative process. Neurotoxicology 25: 555-572.

Cadet JL, Jayanthi S, McCoy MT, Vawter M, Ladenheim B (2001). Temporal profiling of methamphetamine-induced changes in gene expression in the mouse brain: evidence from cDNA array. Synapse 41: 40-48.

Chartoff EH, Marck BT, Matsumoto AM, Dorsa DM, Palmiter RD (2001). Induction of stereotypy in dopamine-deficient mice requires striatal D1 receptor activation. Proc Natl Acad Sci USA 98: 10451-10456.

Eilam R, Davidson A, Gozes I, Segal M (1999). Locomotor activity causes a rapid up-regulation of vasoactive intestinal peptide in the rat hippocampus. Hippocampus 9: 534-541.

Ennulat DJ, Babb Sm, Cohen BM (1994). Persistent reduction of immediate early gene mRNA in rat forebrain following single or multiple doses of cocaine. Brain Res Mol Brain Res 26: 106-112.

Fibiger HC (1991). The dopamine hypotheses of schizophrenia and mood disorders: contradictions and speculations. In: Willner P, Scheel-Kruger J (eds). The Mesolimbic Dopamine System: From Motivation to Action. John Wiley \& Sons Ltd: New York, NY. pp 620-637.

Glickstein SB, Schmauss C (2004). Effect of methamphetamine on cognition and repetitive motor behavior of mice deficient for dopamine D2 and D3 receptors. Ann NY Acad Sci 1025: 110-118.

Gonzalez-Nicolini V, McGinty JF (2002). Gene expression profile from the striatum of amphetamine-treated rats: a cDNA array and in situ hybridization histochemical study. Brain Res Gene Expr Patterns 1: 193-198.

Graybiel AM, Moratalla R, Robertson HA (1990). Amphetamine and cocaine induce drug-specific activation of the c-fos gene in striosome-matrix compartments and limbic subdivisions of the striatum. Proc Natl Acad Sci USA 87: 6912-6916.

Harlan RE, Garcia MM (1998). Drugs of abuse and immediateearly genes in the forebrain. Mol Neurobiol 16: 221-267.

Hooks MS, Colvin AC, Juncos JL, Justice JBJ (1992). Individual differences in basal and cocaine-stimulated extracellular dopamine in the nucleus accumbens using quantitative microdialysis. Brain Res 587: 306-312.

Irizarry RA, Hobbs B, Collin F, Beazer-Barclay YD, Antonellis KJ, Scherf U et al (2003). Exploration, normalization, and summaries of high density oligonucleotide array probe level data. Biostatistics 4: 249-264.

Konradi C, Cole RL, Heckers S, Hyman SE (1994). Amphetamine regulates gene expression in rat striatum via transcription factor CREB. J Neurosci 14: 5623-5634.

Lee KW, Hong JH, Choi IY, Che Y, Lee JK, Yang SD et al (2002). Impaired D2 dopamine receptor function in mice lacking type 5 adenylyl cyclase. J Neurosci 22: 7931-7940.

Lewis DA, Hashimoto T, Volk DW (2005). Cortical inhibitory neurons and schizophrenia. Nat Rev Neurosci 6: 312-324.

Lipska BK, al-Amin HA, Weinberger DR (1998). Excitotoxic lesions of the rat medial prefrontal cortex. Effects on abnormal behaviors associated with neonatal hippocampal damage. Neuropsychopharmacology 19: 451-464.

Moratalla R, Xu M, Tonegawa S, Graybiel AM (1996). Cellular responses to psychomotor stimulant and neuroleptic drugs are abnormal in mice lacking the D1 dopamine receptor. Proc Natl Acad Sci USA 93: 14928-14933.

Ogden CA, Rich ME, Schork NJ, Paulus MP, Geyer MA, Lohr JB et al (2004). Candidate genes, pathways and mechanisms for bipolar (manic-depressive) and related disorders: an expanded convergent functional genomics approach. Mol Psychiatry 9: 1007-1029.

Palmer AA, Verbitsky M, Suresh R, Kamens HM, Reed CL, Li N et al (2005). Gene expression differences in mice divergently selected for methamphetamine sensitivity. Mamm Genome 16: 291-305.

Persico AM, Schindler CW, Zaczek R, Brannock MT, Uhl GR (1995). Brain transcription factor gene expression, neurotransmitter levels, and novelty response behaviors: alterations during rat amphetamine withdrawal and following chronic injection stress. Synapse 19: 212-227.

Saxon AJ, Oreskovich MR, Brkanac Z (2005). Genetic determinants of addiction to opioids and cocaine. Harv Rev Psychiatry 13: $218-232$.

Segal DS, Kuczenski R (1987). Individual differences in responsiveness to single and repeated amphetamine administration: behavioral characteristics and neurochemical correlates. J Pharmacol Exp Ther 242: 917-926.

Segal DS, Kuczenski R (1997). Repeated binge exposures to amphetamine and methamphetamine: behavioral and neurochemical characterization. J Pharmacol Exp Ther 282: 561-573.

Segal DS, Schuckit MA (1983). Animal models of stimulantinduced psychosis. In: Creese I (ed). Stimulants: Neurochemical, Behavioral, and Clinical Perspectives. Raven Press: New York, NY. pp 131-167.

Shilling PD, Kelsoe JR, Kuczenski R, Segal DS (2000). Differential regional zif268 mRNA expression in an escalating dose/binge model of amphetamine-induced psychosis. Neuroscience 96: 83-90.

Sklar P (2002). Linkage analysis in psychiatric disorders: the emerging picture. Annu Rev Genom Hum Genet 3: 371-413.

Sokolov BP, Polesskaya OO, Uhl GR (2003). Mouse brain gene expression changes after acute and chronic amphetamine. J Neurochem 84: 244-252.

Swerdlow NR, Koob GF, Cador M, Lorang M, Hauger RL (1993). Pituitary-adrenal axis responses to acute amphetamine in the rat. Pharmacol Biochem Behav 45: 629-637.

Takaki M, Ujike H, Kodama M, Takehisa Y, Nakata K, Kuroda S (2001). Two kinds of mitogen-activated protein kinase phosphatases, MKP-1 and MKP-3, are differentially activated by acute and chronic methamphetamine treatment in the rat brain. J Neurochem 79: 679-688.

Umemoto S, Kawai Y, Ueyama T, Senba E (1997). Chronic glucocorticoid administration as well as repeated stress affects the subsequent acute immobilization stress-induced expression of immediate early genes but not that of NGFI-A. Neuroscience 80: 763-773.

Wu W, Chaudhuri S, Brickley DR, Pang D, Karrison T, Conzen SD (2004a). Microarray analysis reveals glucocorticoid-regulated survival genes that are associated with inhibition of apoptosis in breast epithelial cells. Cancer Res 64: 1757-1764.

Wu Y, Barrett EJ, Long W, Liu Z (2004b). Glucocorticoids differentially modulate insulin-mediated protein and glycogen synthetic signaling downstream of protein kinase $B$ in rat myocardium. Endocrinology 145: 1161-1166.

Yamagata K, Suzuki K, Sugiura H, Kawashima N, Okuyama S (2000). Activation of an effector immediate-early gene arc by methamphetamine. Ann NY Acad Sci 914: 22-32.

Yuferov V, Nielsen D, Butelman E, Kreek MJ (2005). Microarray studies of psychostimulant-induced changes in gene expression. Addict Biol 10: 101-118. 